\title{
Extended Surgical Resection and Histology in Retroperitoneal Sarcoma
}

\author{
Aimee M. Crago, MD, PhD, FACS \\ Gastric and Mixed Tumor Service, Memorial Sloan Kettering Cancer Center, New York, NY
}

While several decades ago soft tissue sarcoma was often managed as one disease, we now recognize that sarcoma is an umbrella term encompassing almost 100 different pathologies, each characterized by unique genetic aberrations. In many ways, the field of sarcoma biology is on the cutting edge of personalized medicine; we routinely use histologic and molecular information to individualize management algorithms. In this issue of Annals of Surgical Oncology, Gronchi et al. present data collected over a 10year period, and describing outcomes after resection of primary retroperitoneal sarcomas at the Instituto Nazionale Tumori (INT) in Milan and the Institute Gustave Roussy (IGR) in Paris. The study delineates rates of local and distal recurrence as they associate with histology. The results bring up numerous questions regarding our selection of patients for surgery and adjuvant therapy, as well as the manner in which we design and interpret clinical trials seeking to optimize management of retroperitoneal sarcomas.

For example, the results presented by Dr Gronchi and colleagues demonstrate that retroperitoneal leiomyosarcoma has a high propensity for distal recurrence $(55 \%$ of patients in this series with 44 months' follow-up). Very few $(5 \%)$ were observed to have local recurrence without distant recurrence. A similar rate of distal recurrence$43 \%$ at 5 years-was reported by Gladdy et al. ${ }^{1}$ The rate continued to climb over time to $52 \%$ at 10 years after surgery. Data of this nature have significant implications and can alter our approach to retroperitoneal leiomyosarcoma. A $40-50 \%$ distal rate of metastasis is similar to that

(C) Society of Surgical Oncology 2014

First Received: 5 September 2014; Published Online: 15 October 2014

A. M. Crago, MD, PhD, FACS

e-mail: cragoa@mskcc.org observed for large, high-grade extremity tumors. ${ }^{2}$ Patients with these lesions are frequently considered for neoadjuvant/adjuvant chemotherapy. Perhaps patients with retroperitoneal leiomyosarcomas should be considered for similar treatment. Similarly, if risk of local recurrence without distal disease is as low as $5 \%$, then this subgroup has little potential to benefit from neoadjuvant radiation. In fact, one could argue that inclusion of leiomyosarcoma patients in the ongoing EORTC phase III STRASS trial, which examines the role of neoadjuvant radiation, will compromise the power of a study in which the primary endpoint is abdominal recurrence-free survival. ${ }^{3}$

There are limitations to this study. Follow-up is short and, in some histologic subgroups, fewer than ten patients remain to be analyzed 5 years after surgery. Some potentially interesting points are not reported (e.g. do concurrent local and distal recurrences occur in dedifferentiated liposarcoma or leiomyosarcoma?), and it is arguable that patients undergoing $\mathrm{R} 2$ resection should have been excluded from analyses. However, in addition to the description of leiomyosarcoma outcomes discussed above, many other important points are made regarding the role of biology in predicting outcome after resection of retroperitoneal sarcomas. Well-differentiated liposarcomas do not metastasize and, when removed by an experienced surgeon, rates of local control are reasonable. Dedifferentiated liposarcomas are associated with high rates of local recurrence and distal metastases, whereas patients undergoing resection for solitary fibrous tumors generally do very well.

Unfortunately, despite providing useful information, the paper cannot clarify the ongoing debate on whether patients with retroperitoneal sarcomas benefit from extended resection (removal of contiguous but uninvolved organs en bloc with tumor). ${ }^{4,5}$ It seeks to do so given the excellent local recurrence rates achieved by these highvolume surgeons who routinely perform compartmental resection for primary retroperitoneal sarcoma. The basis for 
their argument that routine extended resection should be performed lies in retrospective analyses of tumors treated at the INT during a period when extended resection was routine (2002-2011) versus those managed prior to 2002, and a series of 385 retroperitoneal sarcomas resected at IGR and retrospectively determined to have undergone compartmental (extended) resection compared with simple excision. ${ }^{6,7}$ In both studies, extended resection was associated with improved rates of local recurrence. These results were provocative; however, many arguments have been advanced against the idea that extended resection is superior. For example, the published series of extended resection had short follow-up; extended resection is truly feasible only for the small subset of tumors that do not border great vessels, liver, or diaphragm; retroperitoneal sarcomas rarely invade adjacent structures (e.g. invasion was found in only $9 \%$ of kidneys resected in conjunction with retroperitoneal sarcoma); multivisceral resection carries increased risk of perioperative morbidity; and chronic renal insufficiency may preclude subsequent enrollment in clinical trials. ${ }^{6,8,9}$ In a retrospective review of 177 retroperitoneal liposarcomas, resection of contiguous organs other than the kidney was associated with increased local and distal recurrence, presumably because contiguous organ resection was also associated with larger tumors with more aggressive biology. ${ }^{10}$ Additionally, in the current series but not in the historical controls, the analysis of local recurrence treated distal recurrence as a competing event, a method that may underestimate the true rate of local recurrence.

We have learned an enormous amount regarding the role of surgery in the treatment of retroperitoneal sarcomas over the last 20 years, aided by the development of databases large enough to delineate the biology of these tumors. ${ }^{11} \mathrm{We}$ now recognize that surgical resectability is a predictor of disease-specific survival, ${ }^{12}$ that multifocality means that resection will rarely cure a patient, ${ }^{13}$ and that tumors that recur quickly are unlikely to be effectively treated by surgery. ${ }^{14}$ We know that in liposarcoma patients, normalappearing retroperitoneal fat may actually represent welldifferentiated disease and therefore we consider removing all fatty tissue during tumor resection. From the work of Gronchi et al. published in this edition of Annals of Surgical Oncology, and from other recent reviews, we know that histology predicts outcome. ${ }^{1,10}$ In short, we have significantly improved our ability to identify patients most likely to benefit from surgery, and this may well underlie the observed improvement in patients undergoing extended resection in recent series compared with patients who underwent simple resection over a decade ago.

In fact, Dr. Bonvalot, Dr. Gronchi, and colleagues have presented data that argue this point in their comparison between their recent series of extended resections and

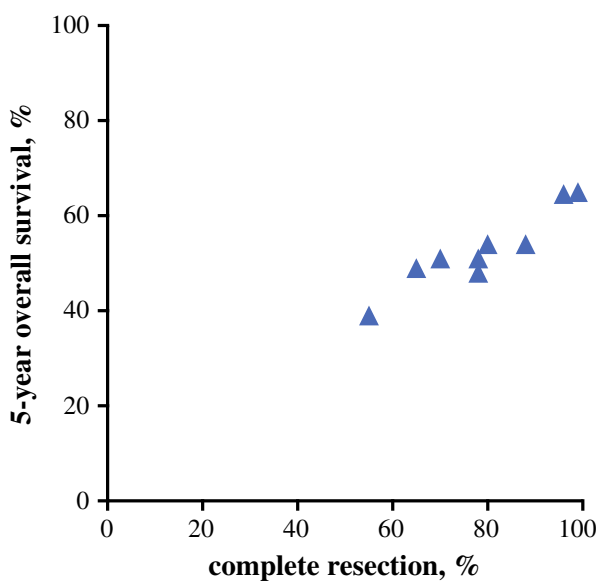

FIG. 1 Rates of complete resection and overall survival at 5 years across eight historic series cited by Bonvalot et al. ${ }^{6}$ and in the current study of Gronchi et al. published in this issue of Annals of Surgical Oncology

historical series. ${ }^{6,7}$ Across the series, absolute differences in 5-year overall mortality were closely correlated with absolute differences in rate of $\mathrm{R} 2$ resection (Fig. 1). 6,7,12,15 These comparisons, as well as histology-based outcomes presented by Gronchi et al., justify judicious use of extended resection. Extended resection may not be justifiable for a disease such as leiomyosarcoma, where rates of distal recurrence are high and local control is unlikely to be the determinant of survival. Extended resection may be more relevant for low-grade lesions such as well-differentiated liposarcomas or grade II liposarcomas, where survival is associated with local control. While outcomes presented by Gronchi et al. raise provocative questions regarding extended resection, associations seen in retrospective series are almost always driven by a bias in patient selection. Only randomized trials or, assuming this fails, carefully designed case-controlled studies specifically designed to address these concerns can provide further insight into the risk-benefit ratio of extended resection.

One last point should be made regarding outcomes in patients with locally recurrent disease after extended resection. In this small series, overall survival following recurrence was particularly dismal compared with prior analyses, and reoperations were rarely indicated. This will clearly take more in-depth review as additional patients are added to the cohort. One can imagine that after extended resection, reoperation would be difficult and recurrences would more often occur at the level of the porta hepatis or central vessels. However, in carefully selected patients, surgical resection of locally recurrent retroperitoneal sarcoma has generally been associated with improved outcomes compared with inoperable tumors. Rarely is it observed, as in this analysis, that outcomes are similar for inoperable patients and those undergoing surgical 
resection. Once the follow-up is longer, a great deal more information will be available on factors influencing patient selection for operation (e.g. multifocality, rate of recurrence) and on which patients do well after surgery; this additional information will clarify treatment algorithms for recurrent disease. We look forward to these reports from our colleagues in the future.

ACKNOWLEDGMENT The author has no disclosures to report.

\section{REFERENCES}

1. Gladdy RA, Qin LX, Moraco N, Agaram NP, Brennan MF, Singer S. Predictors of survival and recurrence in primary leiomyosarcoma. Ann Surg Oncol. 2013;20:1851-7.

2. Eilber FC, Brennan MF, Riedel E, Alektiar KM, Antonescu CR, Singer S. Prognostic factors for survival in patients with locally recurrent extremity soft tissue sarcomas. Ann Surg Oncol. 2005;12:228-36.

3. EORTC. A phase III randomized study of preoperative radiotherapy plus surgery versus surgery alone for patients with retroperitoneal sarcomas (RPS)—STRASS. Available from: http://www.eortc.be/ clinicaltrials/Details.asp?Protocol=62092\&T=. Accessed 22 Aug 2014.

4. Gronchi A, Pollock R. Surgery in retroperitoneal soft tissue sarcoma: a call for a consensus between Europe and North America. Ann Surg Oncol. 2011;18:2107-10.

5. Pisters PW. Resection of some-but not all-clinically uninvolved adjacent viscera as part of surgery for retroperitoneal soft tissue sarcomas. J Clin Oncol. 2009;27:6-8.

6. Bonvalot S, Miceli R, Berselli M, et al. Aggressive surgery in retroperitoneal soft tissue sarcoma carried out at high-volume centers is safe and is associated with improved local control. Ann Surg Oncol. 2010;17:1507-14.

7. Gronchi A, Lo Vullo S, Fiore M, et al. Aggressive surgical policies in a retrospectively reviewed single-institution case series of retroperitoneal soft tissue sarcoma patients. J Clin Oncol. 2009;27:24-30.

8. McKiernan J, Simmons R, Katz J, Russo P. Natural history of chronic renal insufficiency after partial and radical nephrectomy. Urology. 2002;59:816-20.

9. Russo P, Kim Y, Ravindran S, Huang W, Brennan MF. Nephrectomy during operative management of retroperitoneal sarcoma. Ann Surg Oncol. 1997;4:421-4.

10. Singer S, Antonescu CR, Riedel E, Brennan MF. Histologic subtype and margin of resection predict pattern of recurrence and survival for retroperitoneal liposarcoma. Ann Surg. 2003;238:358-70; discussion 370-351.

11. Heslin MJ, Lewis JJ, Nadler E, et al. Prognostic factors associated with long-term survival for retroperitoneal sarcoma: implications for management. J Clin Oncol. 1997;15:2832-9.

12. Lewis JJ, Leung D, Woodruff JM, Brennan MF. Retroperitoneal soft-tissue sarcoma: analysis of 500 patients treated and followed at a single institution. Ann Surg. 1998;228:355-65.

13. Anaya DA, Lahat G, Liu J, et al. Multifocality in retroperitoneal sarcoma: a prognostic factor critical to surgical decision-making. Ann Surg. 2009;249:137-42.

14. Park JO, Qin LX, Prete FP, Antonescu C, Brennan MF, Singer S. Predicting outcome by growth rate of locally recurrent retroperitoneal liposarcoma: the one centimeter per month rule. Ann Surg. 2009;250:977-82.

15. Kilkenny JW 3rd, Bland KI, Copeland EM 3rd. Retroperitoneal sarcoma: the University of Florida experience. J Am Coll Surg. 1996;182:329-39. 